\title{
Demographic and Clinical Factors Predict Focused Shockwave Therapy Results on Patients With Plantar Fasciitis. A Multilevel Analysis
}

\author{
A. Vertuccio ${ }^{1,3}$, D. Perugia ${ }^{1,2}$, R. M. Lanzetti ${ }^{4}$, A. Massimiani ${ }^{1,3}$, A. Lacopo ${ }^{1,3}$, \\ S. M. Nusca ${ }^{1,3}$, R. Baldini ${ }^{1,3}$, F. Santoboni ${ }^{1,3}$, E. Latini ${ }^{1,3}$, M. Vetrano ${ }^{1,3}$, M. C. Vulpiani ${ }^{1,3}$ \\ 1 Department of Surgical and Medical Sciences and Translational Medicine, Faculty of Medicine and Psychology, \\ University of Rome La Sapienza, Rome, Italy \\ 2 Orthopaedic Unit, Sant'Andrea Hospital, University of Rome La Sapienza, Rome, Italy \\ 3 Physical Medicine and Rehabilitation Unit, Sant'Andrea Hospital, University of Rome La Sapienza, Rome, Italy \\ ${ }_{4}$ Orthopaedics and Traumatology Unit, Department of Emergency and Acceptance, San Camillo-Forlanini \\ Hospital, Rome, Italy
}

\section{CORRESPONDING AUTHOR:}

Riccardo Maria Lanzetti

Orthopaedics and Traumatology Unit

Department of Emergency and

Acceptance

San Camillo-Forlanini Hospital

circonvallazione Gianicolense 87

00152 Rome, Italy

E-mail: riccardolanzetti@gmail.com

DOI:

10.32098/mltj.03.2021.02

LEVEL OF EVIDENCE: 4

\begin{abstract}
SUMMARY
Background. Plantar fasciitis (PF) is one of the most common causes of heel pain. The use of ESWT is approved in the treatment of chronic PF. The aim of this study was to conduct a retrospective analysis to create models for a pre-treatment evaluation in people with PF, and to assess whether demographic and clinical features could be used to predict medium-term outcomes six months after fESWT.

Methods. 183 cases of plantar fasciitis were treated with fESWT. The outcome was NRS variation at three and six months. Age, gender, Likert scale and FAOS were the predictive factors evaluated.

Results. A significant improvement in the mean values of NRS and FAOS was observed at the follow up controls. Patients with unfavorable characteristics association (age $\geq$ 56 years, FAOS $<66.7$, Likert scale 3-4) showed higher NRS values, instead patients with favorable characteristics association (age $<56$ years, FAOS $\geq 66.7$, Likert scale 1-2) showed lower NRS values.

Conclusions. The multilevel modeling creates clinical models for a pre-treatment evaluation of patients with PF, predicting a better or worse medium-term after fESWT. The patient presenting a combination of unfavorable characteristics could be informed about a worst therapeutic response, and modification on therapy protocol or association of different therapies could be considered.
\end{abstract}

\section{KEY WORDS}

Plantar fasciitis; ESWT; shock waves; predictive factors; multilevel modeling.

\section{BACKGROUND}

Plantar fasciitis is one of the most common causes of heel pain. Typically, the tender point is located on the plantar fascia origin, at the medial process of the calcaneal tuberosity. The heel pain is worse at the first steps in the morning or after a prolonged period of rest and generally increases with passive stretching of the plantar fascia (1). It affects $7-10 \%$ of the general population and the incidence is $8-21 \%$ among athletes and runners. People aged 40-60 years are mostly affected (2). The aetiology is unclear and probably multifactorial. Plantar fasciitis is generally believed to be due to an overuse injury, when repetitive microtrauma and damage exceed the tissue healing ability, with a chronic inflammation associated. Intrinsic and extrinsic risk factors are recognized: anatomical features (overweight, excessive foot pronation), functional features (Achilles tendon tightness), wearing inadequate footwear, prolonged standing or 
walking occupations, incorrect training (1-3). Patients with plantar fasciitis often present a heel spur, but it also occurs in asymptomatic populations, so its causal role in the development of plantar fasciitis is uncertain (4).

The diagnosis is primarily based on symptoms and clinical evaluation. Plain radiographs frequently reveal heel spur on the inferior surface of the calcaneus, and it is useful to exclude calcaneal stress fracture and other rare bony lesions $(1,3)$. Ultrasound examination is frequently used to confirm the plantar fasciitis diagnosis and exclude other causes of heel pain (1). A hypoechoic and thickened ( $>4$ $\mathrm{mm}$ ) fascia, the presence of bony erosions, heel spur, signs of fascia ruptures and color Doppler activity are the ultrasound aspects that suggest the diagnosis of plantar fasciitis (3). Magnetic resonance (MR) images show the plantar fascia thickening and the T2 high signal intensity area. MR images are often performed for differential diagnosis from other diseases, such as bone marrow edema of the calcaneous. Other differential diagnosis are painful peripheral neuropathies, tarsal tunnel syndrome, lesion of the plantar nerve, tumors, and plantar fascia fibromatosis (4).

Plantar fasciitis is managed conservatively: non-steroidal anti-inflammatory drugs (NSAIDs), corticosteroid injection, foot orthotic devices, physical therapy (laser therapy, cryoultrasound), stretching exercises for foot and lower limb muscles, kinesiotaping, platelet-rich plasma (PRP) injection $(1,3)$. When plantar fasciitis symptoms persist after 6 months of different and repetitive conservative treatments, surgical option is considered. Surgical treatment includes endoscopic or open plantar fascia release and cryosurgery (5). In 2000 the USA Food and Drug Administration (FDA) approved the use of extracorporeal shockwave therapy (ESWT) in the treatment of chronic plantar fasciitis. The rationale for ESWT in clinical use is inhibition of pain receptors and stimulation of soft-tissue healing $(1,3)$. Several studies showed that ESWT induces neo-vascularization in treated tissue and that it is a reparative stimulus through remodeling collagen, reducing the expression of the pro-inflammatory profile M1 macrophages and inducing the expression of an anti-inflammatory profile M2 (6).

Many authors have studied its efficacy on plantar fasciitis and have compared their effects with other conservative treatments $(3,7)$. Yin et al. in their meta-analysis showed a strong statistical evidence of focused ESWT e radial pressure wave efficacy in the treatment of chronic plantar fasciitis in the medium term (within 6 months), with better results using energy flux density $(\mathrm{EFD})<0.20 \mathrm{~mJ} / \mathrm{mm}(2$, 7). Similar results are showed in the more recent meta-analysis of RCT conduct by Li et al. (8). Sun et al. in their meta-analysis analyzed 9 RCT that evaluated the effectiveness of focused ESWT e radial pressure wave in the treat- ment of chronic plantar fasciitis. Both focused ESWT e radial pressure waves were more effective than placebo, with better results for focused ESWT (9). ESWT has been widely used as a treatment option for plantar fasciitis due to its non-invasive feature, fast recovery time and convenience for daily life of patients (10). In literature, demographic, clinical and ultrasound factors have been investigated as predictors of a better response to ESWT, with conflicting results (11-17). The aim of this study was to conduct a retrospective analysis to create models for a pre-treatment evaluation in people with plantar fasciitis, and to assess whether demographic and clinical features could be used to predict medium-term outcomes six months after focused ESWT.

\section{MATERIALS AND METHODS}

\section{Study design and setting}

From January 2015 to March 2018 in the Physical Medicine and Rehabilitation unit of S. Andrea Hospital in Rome (Sapienza University) a total of 691 cases of plantar fasciitis were treated with focused ESWT. All participants provided hand-signed written informed consent to use collected data for retrospective clinical research purposes. Furthermore, patient data were anonymized and de-identified prior to the analysis. Patients who underwent a clinical examination before the treatment and had two clinical follow-up controls ( 3 and 6 months after the treatment) were considered eligible for the study. Criteria of exclusion was diagnosis other than plantar fasciitis (e.g., bone edema in MRI images, neuropathies). The study meets the ethical standards of Muscles, Ligaments and Tendons Journal (18).

\section{Equipment}

An electromagnetic generator (Modulith ${ }^{\circledR}$ SLK Storz Medical) with in-plane ultrasound guide was used to carry out the treatment: the focus was on the calcaneal enthesis. Every patient received 3 sessions of focused ESWT, once a week. At each session, patient received 2400 shocks, at frequency $4 \mathrm{~Hz}$, at energy flux density (EFD) between 0.14 and $0.20 \mathrm{~mJ} / \mathrm{mm}^{2}$, according to literature data (19) and based on patient's pain tolerance. No local anesthesia was practiced. At the end of the treatment patients were advised to perform home-based stretching exercises of the plantar fascia to practice daily for at least 30 days.

\section{Outcome measures}

The outcome is expressed in terms of variation of the dependent variable Numerical Rating Scale of pain (NRS). NRS is 
a segmented numeric version of the visual analogue scale (VAS) in which the patient selects a whole number $(0-10$ integers) that best reflects the intensity of his pain, with 0 representing "no pain" and 10 representing "pain as bad as you can imagine" and "worst pain imaginable". In this study, NRS refers to the first few steps in the morning and was evaluated before the treatment and at the follow-up controls (at 3 and at 6 months).

Predictive factors evaluated were the following independent variables: age, gender, severity of the disease (Likert scale), Foot and Ankle Outcome Score (FAOS) and time. The severity of the disease was evaluated through a Likert five-points scale (0: no pain; 1: pain in sport and intense activities, no limitation; 2: pain, stiffness in the morning and limitation of intense activities; 3 : pain and limitation in daily activities; 4: pain at rest, continuous) (20). The Foot and Ankle Outcome Score (FAOS) is a patient-reported questionnaire measuring symptoms and functional limitations of the foot and ankle. The FAOS consists of 42 questions, divided into five different patient-relevant subscales: pain; symptoms such as stiffness, swelling and range of motion; activities of daily living; ability to perform sports and recreational activities; foot/ankle-related quality of life. The total score is recorded into a 0-100 scale, with 100 representing no symptoms or limitations (21). For the statistical analysis, only the total score was considered. Likert scale and FAOS were evaluated before the treatment and at the follow-up controls (at 3 and at 6 months).

\section{Statistical analysis}

Continuous variables were described using the average value and standard deviation. Repeated measures ANOVA was used to compare the average NRS and FAOS values at baseline and at the two follow-up control, at three and six months. A multilevel modeling was used to evaluate the relationship between the outcome dependent variable of interest (NRS) and the predictive independent variables under study (age, gender, severity of the disease as Likert scale, Foot and Ankle Outcome Score FAOS, time), considering the correlated data structure and the nesting of observations. Multilevel modeling was used for data analysis as it adequately represents the unexplained variability of the nested structure, which is often arduous to describe in the single-level approach. The combination of the predictor factors at $\mathrm{T}_{0}, \mathrm{~T}_{1}$ and $\mathrm{T}_{2}$ led to the creation of patient models that showed better or poorer clinical results. A threshold of significance was considered at p-value $<0.05$. Statistical analysis was conducted using StataSE-12.

\section{RESULTS}

Of the 691 cases treated with focused ESWT, 183 cases were eligible for the study (figure 1). The male patients were 110 and the female patients were 73 . The average age was 56 years.

A significant improvement in the mean values of NRS and FAOS was observed at the follow-up controls, at three and six months after treatment (table I).

The multilevel modeling analyzed the statistically significant independent variables as predictive factors: based on time, NRS decreased with a coefficient of -1.7 (p-value $<0.001$ );

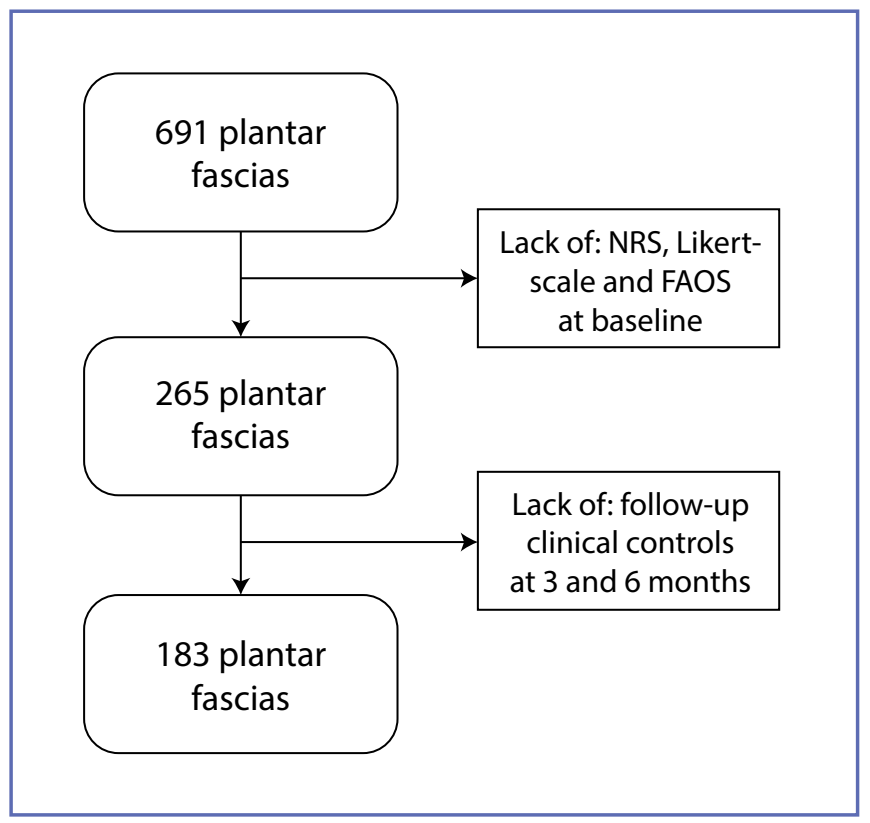

Figure 1. Enrolled patients.

Table I. Average NRS and FAOS values.

\begin{tabular}{lcccc} 
& $\mathbf{T}_{0}$ & $\mathbf{T}_{1}$ & $\mathbf{T}_{2}$ & p-value \\
\hline $\begin{array}{l}\text { NRS } \\
\text { (average } \pm \text { SD) }\end{array}$ & $6.5 \pm 1.6$ & $4.0 \pm 2.2$ & $2.9 \pm 2.5$ & 0.000 \\
\hline $\begin{array}{l}\text { FAOS } \\
\text { (average } \pm \text { SD) }\end{array}$ & $64.4 \pm 14$ & $80.2 \pm 15.6$ & $85.9 \pm 15.5$ & 0.000 \\
\hline
\end{tabular}


based on FAOS, NRS decreased with a coefficient of - 1.4 ( $p$-value $<0.001$ ); based on Likert scale, NRS increased with a coefficient of 1.5 (p-value $<0.001$ ); based on the age, NRS increased with a coefficient of 0.33 (p-value $=0.052$ ). Gender was not considered because it was not statistically significant ( $\mathrm{p}$-value $=0.666)$. Age was considered as predictive factor because it tended to statistically significant $(p$-value $=0.052)$ (table II).

The significant variables were dichotomized on the median value to create clinical models: age $<56$ years and $\geq 56$ years; FAOS $<66.7$ and $\geq 66.7$; Likert scale 1-2 and 3-4. Higher NRS values are observed in patients with more unfavorable characteristics association, that are age $\geq 56$ years, FAOS $<$ 66.7 and Likert scale 3-4. Lower NRS values are observed in patients with more favorable characteristics association, that are age $<56$ years, FAOS $\geq 66.7$, and Likert scale 1-2. Likert scale seems to be the most discriminating variable for the variation of NRS at three months and at six months, lesser FAOS (table III).

\section{DISCUSSION}

A multilevel modeling was used to create clinical models for a pre-treatment evaluation predicting medium-term outcomes six months after focused ESWT. The study results have shown that higher NRS values are expected in patients with more unfavorable characteristics association: age $\geq 56$ years, FAOS $<66.7$ and Likert scale 3-4. Instead, lower NRS values are expected in patients with more favorable characteristics association: age $<56$ years, FAOS $\geq 66.7$ and Likert scale 1-2. Likert scale seems to be the most discriminating variable for the variation of NRS at three and six months, lesser FAOS. Despite the retrospective nature of the study and the monocentric acquisition of data, the effects of potential confounders were adjusted in the multilevel modeling, which takes into account the correlated data structure and the nesting of observation. Through the combination of the predictive factors (age, FAOS and Likert scale), this statistical analysis presented different typologies of patient, with

Table II. Regression coefficients of the independent variables.

\begin{tabular}{llll} 
& Coeff. & p-value & IC (95\%) \\
\hline Time & -1.7 & 0.000 & $-1.88 ;-1.59$ \\
\hline FAOS & -1.4 & 0.000 & $-1.68 ;-1.08$ \\
$\leq 66.7$ & & & \\
$>66.7$ & 1.5 & 0.000 & $1.16 ; 1.80$ \\
\hline Likert-scale & & & \\
I-II & & 0.052 & $-0.003 ; 0.65$ \\
III-IV & 0.33 & & \\
\hline $\begin{array}{l}\text { Age } \\
\leq 56\end{array}$ & & 0.666 & $-0.82 ; 0.46$ \\
$>56$ & -0.09 & & \\
\hline $\begin{array}{l}\text { Gender } \\
\text { Male }\end{array}$ & & & \\
Female & & & \\
\hline
\end{tabular}

CI: confidence interval.

Table III. Clinical models created by the multilevel modeling.

\begin{tabular}{llllll}
\hline \multicolumn{2}{l}{ CLINICAL MODELS } & & NRS baseline & $\begin{array}{l}\text { NRS } \\
\mathbf{3} \text { months }\end{array}$ & $\begin{array}{l}\text { NRS } \\
\mathbf{6} \text { months }\end{array}$ \\
\hline Age $\geq 56$ & FAOS $<66.7$ & Likert scale 3-4 & 6.7 & 5.7 & $\mathbf{5 . 7}$ \\
\hline Age $<56$ & FAOS $<66.7$ & Likert scale 3-4 & 8.0 & 5.4 & $\mathbf{4 . 5}$ \\
\hline Age $\geq 56$ & FAOS $\geq 66.7$ & Likert scale 3-4 & 7.0 & 4.4 & 3.5 \\
\hline Age $<56$ & FAOS $\geq 66.7$ & Likert scale 3-4 & 6.7 & 4.1 & 3.2 \\
\hline Age $\geq 56$ & FAOS $<66.7$ & Likert scale 1-2 & 6.7 & 4.1 & 3.2 \\
\hline Age $<56$ & FAOS $<66.7$ & Likert scale 1-2 & 6.4 & 3.8 & $\mathbf{2 . 9}$ \\
\hline Age $\geq 56$ & FAOS $\geq 66.7$ & Likert scale 1-2 & 5.4 & 2.8 & $\mathbf{1 . 9}$ \\
\hline Age $<56$ & FAOS $\geq 66.7$ & Likert scale 1-2 & 5.1 & 2.5 & $\mathbf{1 . 6}$ \\
\hline
\end{tabular}


better or worse outcome to the treatment, that could guide clinical decisions. The lack of other clinical predictors, such as body mass index (BMI), pain duration or sport activity, as reported in other studies, is due to the retrospective study design. Moreover, no other diagnostic data were considered (e.g., $\mathrm{Rx}$ for calcaneal spur detection). In our clinical practice, patients underwent an ultrasound evaluation before treatment and 3 month after ESWT, however ultrasound parameters were not included in the modeling for the lack of a 6-months ultrasound follow-up and multilevel analysis requires at least two follow up controls.

In literature, many demographic and clinical features were examined as prognostic factors on the treatment outcome, although to our knowledge none of these have used multilevel modeling. Hansen et al. conducted a cohort study on 174 patients with PF treated by ultrasound-guided corticosteroids injection, to evaluate the long-term prognosis. They found that the female gender and bilateral calcaneal pain showed a significantly elevated risk of having long-term symptoms. Instead, age, BMI, smoker state, physical activity, symptoms induced by physical exercise, fascia thickness and presence of the calcaneal spine did not seem to have any impact on the prognosis (2). Fleischer et al. carried out a secondary analysis of the data obtained from a double-blind RCT, in which demographic and clinical characteristics were investigated as predictive of response to conservative therapy for plantar fasciitis. Age showed a moderate negative association with pain, while BMI showed a moderate positive association with pain and disability. Instead, laterality of symptoms, the duration of symptoms, the self-reported standing time and fascia thickness were not associated with pain or disability (22).

The relationship between the characteristics of the patient with plantar fasciitis and the outcome of treatment with ESWT is still controversed. Notarnicola et al. conducted an observational study whose purpose was to find epidemiological and clinical prognostic factors that could influence the outcome of patients treated with focused ESWT. They evaluated 355 patients with tendinopathies (rotator cuff tendinopathy, epicondylitis, achilles tendinopathy, trochanteritis, jumper's knee) and plantar fasciitis (135 plantar fasciitis) treated with focused ESWT. Predictors of therapeutic benefit were the male gender and high BMI. The other examined features were not predictive (age, diet, blood group, work or sport, comorbidity, drugs, type of tendinopathy, other physiotherapy treatments, applied EFD). The patients with pathology of the dominant limb seem to have more possibilities to limit functional damage (11). Notarnicola conducted also a prospective observational cohort study to verify the clinical outcomes after focused ESWT in patients affected by plantar fasciitis with and without spur, and found that patients report a clinical improvement regardless of calcaneal spur presence or not (15). Other studies examined calcaneal spur presence as predictive for outcome after ESWT, with contrasting results. According to Ermutlu et al., the presence of calcaneal spur was not predictive for functional recovery, like either age, fascia thickness, and duration of symptoms. AOFAS scores was the only predictive factor (16). In Chuckpaiwong cohort study, plantar heel spur did not have a statistically significant effect on the outcome of ESWT, as well as other factors (gender, marital status, BMI, amount of exercise, previous cortisone injections, duration of symptoms, bilateral symptoms, bilateral treatment, smoking, plantar fascia thickness and associated foot pathology). In the same study, the authors conducted a multivariable statistical analysis to assess factors leading to successful outcomes. They found that age, walking more than 8 hours per day, diabetes mellitus, and documented psychological disorder significantly affected the outcome (17). Liang et al. studied the correlation between fascia thickness and pain after treatment with focal ESWT, and stated that patients with thinner plantar fascia experienced less pain after treatment (12). Yin et al. tried to identify the predictive factors for achieving the minimum clinical success of the therapy (MCST, a minimal reduction of $50 \%$ in the VAS score), in order to construct a predictive model for the application of radial pressure waves therapy. They conducted a randomized controlled trial on 278 patients, and the variables considered were: age, gender, BMI, affected side (unilateral or bilateral), duration of symptoms, Roles and Maudsley score, VAS score (pain evoked in the morning after the first steps), edema, calcaneal spur and EFD applied. Using a multivariate logistic regression model, in the three-month follow-up from treatment they identified three predictors of efficacy of radial pressure waves: higher VAS score, edema and calcaneal spur. No effect was found on the response to shockwave treatment for the other variables, although the patients with history of long-standing plantar fasciitis seem to be less responsive to treatment (13). According to this last statement is the work of Saxena et al., which states that early treatment of plantar fasciitis with radial pressure waves, within 3 months of the onset of symptoms, is more likely to allow for maintenance of patients' activity level, instead waiting 6 months to treat plantar fasciitis results in delays and inferior results (23). Yin et al. recently conducted also a cohort study on 210 patients. Using "Artificial neural networks" (ANN) algorithm, the authors created a multilayer model to identify the predictive factors for a minimum clinically successful therapy (MCST) six months after radial pressure waves for chronic plantar fasciitis. The results revealed that the VAS score before ESWT was the most influential factor deciding the odds of successful MCST. Higher VAS, short-duration 
severer pain on taking first steps in the morning and calcaneal spur are important prognostic factors for the efficacy of radial pressure waves. Moreover, high BMI was a prognostic factor positively influencing the clinical efficacy of ESWT. Other factors, such as age, gender, affected bilateral side, Roles and Maudsley score, intensity grade and edema, were not predictive (14).

The knowledge of the prognostic factors leads physiatrist, orthopedic surgeon and physiotherapist to better understand the extent of recovery, consequently adapting the rehabilitation path and the patient's expectations. In this study the multilevel modeling provides different patient typologies combining the predictive factors age, FAOS and Likert scale, based on time. The results of this statistical analysis make it possible to predict a lower response to treatment in patients presenting a combination of unfavorable characteristics (age above 56 years, greater degree of pain and minor degree of function) at the pre-treatment evaluation. These patients should be informed about possible delays in healing, and modification on therapy protocol or association of different therapies could be considered. A second cycle of ESWT could lead the patient to recovery, although a great number of sessions seems to be linked to a higher rate of therapeutic failure, so patients unresponsive after two or more ESWT cycles should be directed to other types of treatment (11). Stretching exercises of the posterior muscle chain, in association with ESWT, may provide better results in terms of pain relief and functional improvement (24). A patient with unfavorable pre-treatment characteristics could benefit from a specific stretching exercises protocol to perform daily, in association to ESWT, as we recommend to our patients from 2018. Recently Notarnicola $e t$ al. studied the effect of a combined protocol, applying focal ESWT to the plantar fascia and muscle mass of gastrocnemius in the same session, thus obtaining better results in terms of recovery of dorsal and plantar flexion (25-29). Similar results were obtained by Giordani et al., whose treatment protocol required that the therapy was focused on the active trigger or myofascial points of the leg, thigh e pelvis in order to restore the correct plantar fascia function, the sliding properties and the equilibrium in the myofascial system of the entire lower limb (6). Modifying the treatment protocol by applying focal ESWT to other points, in addition to the standard calcaneal enthesis point of the plantar fascia, could improve clinical results in patients with unfavorable pre-treatment characteristics. Further studies using the multilevel modeling are necessary to increase the patients' sample. Moreover, other predicting factors, such as BMI, pain duration, sport activity, ultrasound parameters or presence of calcaneal spur, should be considered to improve modeling accuracy and to better target clinicians' work. It would be interesting to evaluate combined therapy protocols to improve recovery for those patients who present association of negative prognostic factors at the time of plantar fasciitis diagnosis.

\section{CONCLUSIONS}

The multilevel modeling allows us to create clinical models for a pre-treatment evaluation of patients with plantar fasciitis, predicting a better or worse medium-term outcome six months after focused ESWT. Therefore, it could be possible to predict a lower response to treatment in patients presenting a combination of unfavorable characteristics (age above 56 years, greater degree of pain and function) at the pre-treatment evaluation. These patients could be informed about a worst therapeutic response, and modification on therapy protocol or association of different therapies could be considered.

\section{ACKNOWLEDGMENTS}

The authors thank Prof. Rossella Baldini for statistical analysis.

\section{CONFLICT OF INTERESTS}

The authors declare that they have no conflict of interests.

\section{REFERENCES}

1. Petraglia F. Plantar fasciitis in athletes: diagnostic and treatment strategies. A systematic review. Muscle Ligaments Tendons J 2017;7(1):107.

2. Hansen L, Krogh TP, Ellingsen T, Bolvig L, Fredberg U. Long-Term Prognosis of Plantar Fasciitis: A 5- to 15-Year Follow-up Study of 174 Patients With Ultrasound Examination. Orthop J Sport Med 2018;6(3):2325967118757983.

3. Rompe JD, Furia J, Weil L, Maffulli N. Shock wave therapy for chronic plantar fasciopathy. Br Med Bull 2007;8182(1):183-208.

4. Jessup R, Oates M, Johnston R, Buchbinder R. Shockwave therapy for plantar heel pain (plantar fasciitis). Cochrane Database Syst Rev 2019;(11):1465-858.

5. Çatal B, Bilge A, Ulusoy RG. Endoscopic Plantar Fascia Release versus Cryosurgery for the Treatment of Chronic Plan- 
tar Fasciitis: A Prospective Randomized Study. J Am Podiatr Med Assoc 2020;110(5):Article_3.

6. Giordani F, Bernini A, Müller-Ehrenberg H, Stecco C, Masiero S. A global approach for plantar fasciitis with extracorporeal shockwaves treatment. Eur J Transl Myol 2019;29(3):171-7.

7. Yin M, Ye J, Yao M, et al. Is Extracorporeal Shock Wave Therapy Clinical Efficacy for Relief of Chronic, Recalcitrant Plantar Fasciitis? A Systematic Review and Meta-Analysis of Randomized Placebo or Active-Treatment Controlled Trials. Arch Phys Med Rehabil 2014;95(8):1585-93.

8. Li S, Wang K, Sun H, et al. Clinical effects of extracorporeal shock-wave therapy and ultrasound-guided local corticosteroid injections for plantar fasciitis in adults. Medicine (Baltimore) 2018;97(50):e13687.

9. Sun J, Gao F, Wang Y, Sun W, Jiang B, Li Z. Extracorporeal shock wave therapy is effective in treating chronic plantar fasciitis. Medicine (Baltimore) 2017;96(15):e6621.

10. Crawford F, Thomson C. Interventions for treating plantar heel pain. Cochrane database Syst Rev 2003;11(3):CD000416.

11. Notarnicola A, Maccagnano G, Tafuri S, et al. Prognostic factors of extracorporeal shock wave therapy for tendinopathies. Musculoskelet Surg 2016;100(1):53-61.

12. Liang HW, Wang TG, Chen WS, Hou SM. Thinner plantar fascia predicts decreased pain after extracorporeal shock wave therapy. Clin Orthop Relat Res 2007;PAP(460):219-25.

13. Yin M, Chen N, Huang Q, et al. New and Accurate Predictive Model for the Efficacy of Extracorporeal Shock Wave Therapy in Managing Patients With Chronic Plantar Fasciitis. Arch Phys Med Rehabil 2017;98(12):2371-7.

14. Yin $\mathrm{M}, \mathrm{Ma} \mathrm{J}, \mathrm{Xu} \mathrm{J}$, et al. Use of artificial neural networks to identify the predictive factors of extracorporeal shock wave therapy treating patients with chronic plantar fasciitis. Sci Rep 2019;9(1):4207.

15. Notarnicola A, Maccagnano G, Moretti L, et al. Could the presence of heel spur be a prognostic factor for outcome of extracorporeal shock wave therapy for plantar fasciitis? J Biol Regul Homeost Agents 2019;33(6):1949-54.

16. Ermutlu C, Aksakal M, Gümüştaş A, Özkaya G, Kovalak E, Özkan Y. Thickness of plantar fascia is not predictive of functional outcome in plantar fasciitis treatment. Acta Orthop Traumatol Turc 2018;52(6):442-6.

17. Chuckpaiwong B, Berkson EM, Theodore GH. Extracorporeal Shock Wave for Chronic Proximal Plantar Fasciitis: 225 Patients with Results and Outcome Predictors. J Foot Ankle Surg 2009;48(2):148-55.

18. Padulo J, Oliva F, Frizziero A, Maffulli N. Muscles, ligaments and tendons journal - Basic principles and recommendations in clinical and field science research: 2016 update. Muscles Ligaments Tendons J 2018;8(3):305-7.

19. Wang YC, Chen SJ, Huang PJ, Huang HT, Cheng YM, Shih CL. Efficacy of Different Energy Levels Used in Focused and Radial Extracorporeal Shockwave Therapy in the Treatment of Plantar Fasciitis: A Meta-Analysis of Randomized Placebo-Controlled Trials. J Clin Med 2019;8(9):1497.

20. Guyatt GH, Townsend M, Berman LB, Keller JL. A comparison of Likert and visual analogue scales for measuring change in function. J Chronic Dis 1987;40(12):1129-33.

21. Roos EM, Brandsson S, Karlsson J. Validation of the foot and ankle outcome score for ankle ligament reconstruction. Foot ankle Int 2001;22(10):788-94.

22. Fleischer AE, Albright RH, Crews RT, Kelil T, Wrobel JS. Prognostic value of diagnostic sonography in patients with plantar fasciitis. J Ultrasound Med 2015;34(10):1729-35.

23. Saxena A, Hong BK, Yun AS, Maffulli N, Gerdesmeyer L. Treatment of Plantar Fasciitis With Radial Soundwave "Early" Is Better Than After 6 Months: A Pilot Study. J Foot Ankle Surg 2017;56(5):950-3.

24. Rompe JD, Furia J, Cacchio A, Schmitz C, Maffulli N. Radial shock wave treatment alone is less efficient than radial shock wave treatment combined with tissue-specific plantar fascia-stretching in patients with chronic plantar heel pain. Int J Surg 2015;24:135-42.

25. Notarnicola A, Maccagnano G, Farì G, et al. Extracorporeal shockwave therapy for plantar fasciitis and gastrocnemius muscle: effectiveness of a combined treatment. J Biol Regul Homeost Agents 2020;34(1):285-90.

26. Lou J, Wang S, Liu S, Xing G. Effectiveness of Extracorporeal Shock Wave Therapy Without Local Anesthesia in Patients With Recalcitrant Plantar Fasciitis: A Meta-Analysis of Randomized Controlled Trials. Am J Phys Med Rehabil 2017;96(8):529-34.

27. Petraglia F, Ramazzina I, Costantino C. Plantar fasciitis in athletes: diagnostic and treatment strategies. A systematic review. Muscles Ligaments Tendons J 2017;7(1):107-18.

28. Maffulli G, Padulo J, Iuliano E, Saxena A, Rompe J. Maffulli $N$ Extracorporeal shock wave therapy in the management of insertional plantar fasciitis: the ASSERT database. Muscles Ligaments Tendons J 2018;8:402-8.

29. Saxena A, Fournier M, Gerdesmeyer L, Gollwitzer H. Comparison between extracorporeal shockwave therapy, placebo ESWT and endoscopic plantar fasciotomy for the treatment of chronic plantar heel pain in the athlete. Muscles Ligaments Tendons J 2013;2(4):312-6. 